This item was submitted to Loughborough's Research Repository by the author.

Items in Figshare are protected by copyright, with all rights reserved, unless otherwise indicated.

\title{
Modelling without queues: adapting discrete-event simulation for service operations
}

PLEASE CITE THE PUBLISHED VERSION

http://dx.doi.org/10.1057/jos.2014.21

\section{PUBLISHER}

Palgrave Macmillan (@ Operational Research Society Ltd)

\section{VERSION}

AM (Accepted Manuscript)

\section{PUBLISHER STATEMENT}

This work is made available according to the conditions of the Creative Commons Attribution-NonCommercialNoDerivatives 4.0 International (CC BY-NC-ND 4.0) licence. Full details of this licence are available at: https://creativecommons.org/licenses/by-nc-nd/4.0/

\section{LICENCE}

CC BY-NC-ND 4.0

\section{REPOSITORY RECORD}

Robinson, Stewart. 2019. "Modelling Without Queues: Adapting Discrete-event Simulation for Service Operations". figshare. https://hdl. handle.net/2134/16839. 


\title{
MODELLING WITHOUT QUEUES: ADAPTING DISCRETE-EVENT SIMULATION FOR SERVICE OPERATIONS
}

\begin{abstract}
Discrete-event simulation, which has largely grown out of modelling manufacturing systems, has increasingly been applied in the service sector. The approach, however, is not always appropriate for modelling service operations. In particular, it cannot help with detailed decisions about the layout of service operations in which the customers are present such as retail outlets and airports. An adapted discrete-event simulation approach is proposed for modelling such systems and the approach is demonstrated through a model of a coffee shop. A key innovation is that queues are not explicitly modelled. The benefit of the approach is that it simplifies the modelling of service systems in which the customers are present by reducing the number of components that need to be modelled. It can also aid decisions about the layout of a system. We ask whether the approach is in fact an agent-based simulation and identify ways in which the approach could be extended.
\end{abstract}

Keywords: Simulation, Service Operations Layout, Discrete-Event Simulation, Agent-Based Simulation 


\section{INTRODUCTION}

The early developments in discrete-event simulation (DES) were largely founded on the manufacturing sector (Tocher, 2008). These models follow a basic structure of active and dead states where entities move from activities to queues, often alternating between these two types of component (Paul, 1993). Activities typically represent steps in the manufacturing process such as machines or manual assembly and queues are used to model buffering between those steps, for instance, conveyors or storage racks. This is a natural representation for a process where the flow of entities is strictly controlled.

In more recent years DES has been applied to the service sector to model, for instance, supermarkets, airports and call centres. There are, however, some key differences between manufacturing and service operations, not least that customers take part in the service process and so the service is 'co-created' or 'co-produced' with the customer (Johnston et al, 2012). As such, from the customers' perspective, the service is the experience received which leads to an outcome (product, benefit, intention).

In transferring the ideas of DES from the manufacturing sector the same basic modelling approach has been adopted, that is, representing service systems as a set of alternating activities and queues. This has been done with some level of success; see for instance Beck (2011) on modelling passenger flow in an airport terminal, and Robinson (2001) on modelling a customer help centre. There are, however, two key differences between modelling service operations where the customers are physically present (e.g. retail outlets or airport terminals) and modelling a manufacturing system. First, the entities (customers) are autonomous and so they cannot be strictly controlled. As such, the sequence of activities a customer follows is in part determined by the customer and the way in which a customer moves from activity to activity is also subject to his/her own behaviour. Second, the customers' experience in the service system is important in determining their level of satisfaction. This suggests that we need to adapt the DES approach for modelling service operations where the customers are present to model customer behaviour in the system and to measure their experience of the system.

Further to this, whereas it may be convenient to have transferred the DES modelling approach from manufacturing to the service sector, it may not be fully effective in addressing 
the issues of the new sector. Indeed, the author's experience of applying DES to service operations has shown that it may not always be able to meet the objectives of the clients. More specifically, as the example in section 2 demonstrates, normal DES is not able to help with detailed facility layout decisions for service operations in which the customers are present. As such, we may need to look for an alternative modelling approach to help in these decision situations.

In this paper an adapted DES approach is proposed in which customers are modelled at an individual level. This enables issues of facility layout to be addressed. The aim is to devise an easy-to-use modelling environment in which alternative layouts can be compared for their effect on the dynamics of a service system in which the customers are present. This will enable a user to determine not only the quantity of service points required, but also their position. A key feature of the proposed approach is that queues do not need to be explicitly modelled, which could make the modelling of the system simpler (section 5.1). Instead, queues emerge as a result of the dynamics of the system being modelled.

We first discuss the limitations of DES models of service systems in which the customers are present through the use of a previous example of modelling a coffee shop. We then discuss previous work on adapting DES for modelling service systems and for modelling human movement, which is a central requirement for modelling service systems in which the customers are present. The proposed approach is demonstrated through an alternative model of a coffee shop that is implemented in Excel/VBA. The discussion that follows identifies the differences between the proposed modelling approach and previous models of such service systems, and it identifies the benefits of the adapted DES. It also discusses whether the proposed approach is in fact an agent-based simulation (ABS); a model composed of autonomous, interacting agents (Macal and North, 2010). Finally, the ways in which the model might be extended are discussed.

\section{DES MODELS OF SERVICE OPERATIONS IN WHICH THE CUSTOMERS ARE PRESENT}

The story of this paper begins around ten years ago with two separate attempts to develop DES models of retail outlets. Both projects were performed by Masters students as the basis of their dissertation. The first project was a simulation for an optician's chain which aimed to 
help determine the facilities required in a specific high street outlet. The second project had a similar aim, but was carried out with a bakery/coffee shop chain. In both cases we developed a typical DES model that included customer arrival rates, queues, service points and, in the case of the bakery, the tables which are used by some customers for consuming their food (figure 1). In these models the customers moved alternately from activities to queues in much the same manner as entities in a manufacturing simulation.

Figure 1 DES Model of Bakery/Coffee Shop

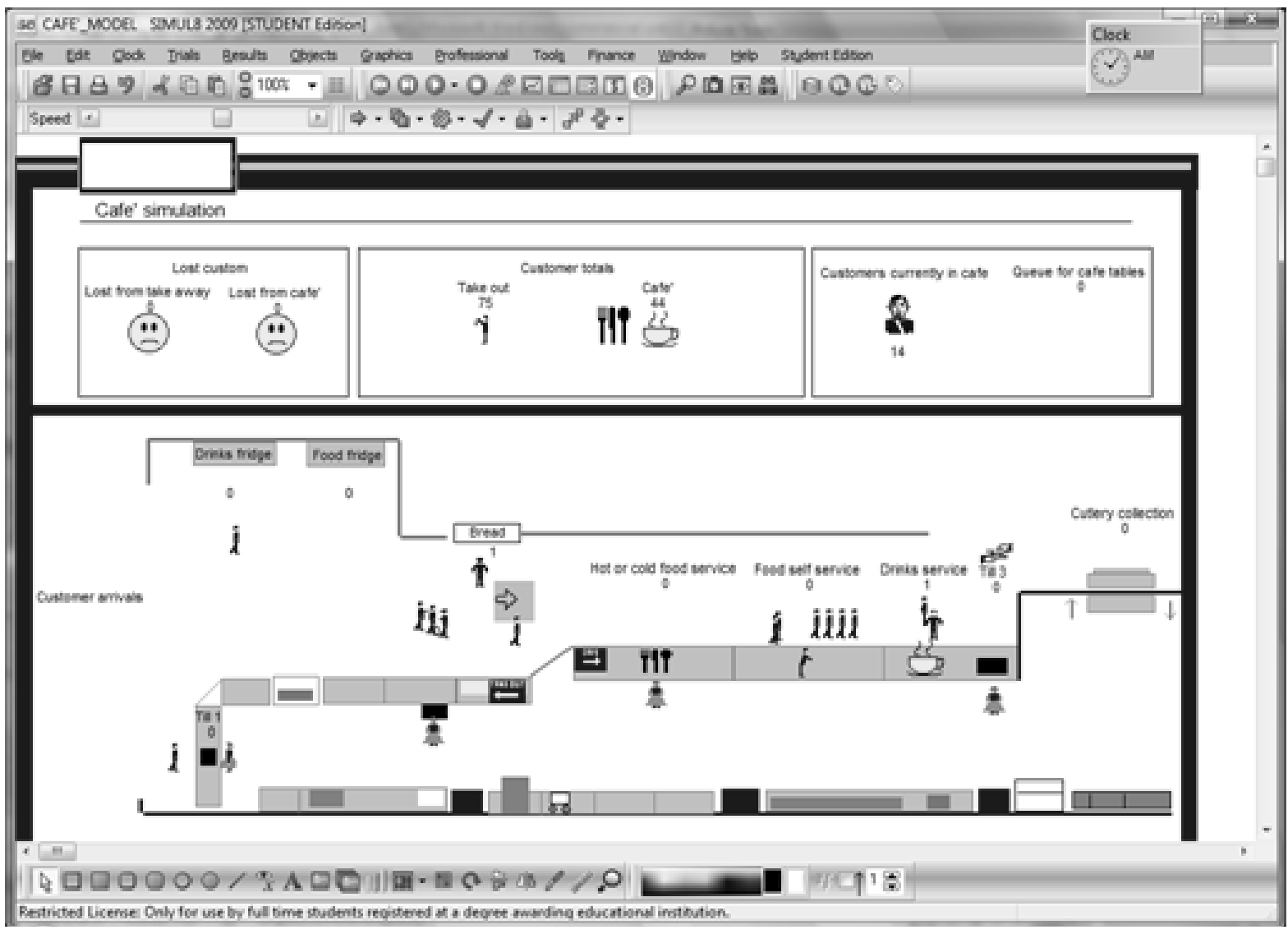

At one level both projects were successful. We were able to determine the number of service points required to meet different levels of customer demand. At another level the work was not so successful and in both instances the work did not progress beyond the period of the Masters project. This was because the question of how many service points are needed was not particularly vexed. These organisations had much experience of managing their high street operations and, given the limited space available to them in any store, they were generally able to determine the appropriate number of resources. It was a comment late on in the project from a manager at the bakery chain that made us aware of the real issue: 'what 
your models can't tell me is where to put the resources.' It was then apparent that the bakery chain needed help in designing the layout of a store and not so much with determining what to put in the store. They wanted to understand the effect of the layout on the flow of customers around the relatively small space available for providing the services required. For instance, what if they moved the cold drinks fridge from one location to another? How would this affect the dynamics of the store?

Our DES models were not able to answer these questions. We could make the model look like a specific layout, but moving a fridge would at most change the travel time to get to and from that fridge. It would not materially affect the dynamics of the model, at least, not without extensive coding every time a layout change was made. This is because wherever the fridge was located we were still assuming that the customers would behave in the same manner through the network of alternating activities and queues. In the real system, however, moving the cold drinks fridge might make the fridge more or less accessible. This could affect the flow of customers around the outlet and might even affect their purchasing decisions. Indeed, the performance of a service operation depends in part on the layout of that operation (Pagell and Melnyk, 2004).

Questions of layout are not just restricted to small retail outlets. Supermarkets face the same issue when designing stores and determining where to place stock. The author has also been part of discussions on modelling the design of a major international airport terminal where issues of layout and the effect on passenger flow have been of great importance.

\section{SIMULATING SERVICE OPERATIONS LAYOUT: ADAPTING DES AND MODELLING HUMAN MOVEMENT}

Given that questions of layout are important and that traditional DES models are not particularly suited to answering such questions, an alternative modelling approach is required. We now explore previous work on simulation which has specifically addressed this issue. First we focus on ways in which DES has been adapted to model service operations layout. We then focus on modelling human movement since this is central to representing the flow of customers around a service system. 


\subsection{Adapting DES and Using ABS for Modelling Service Operations Layout}

Literature research has so far found only three examples of either adapting DES or using ABS for modelling the layout of service operations. Dubiel and Tsimhoni (2005) integrate ABS into DES to model a theme park; a service operation involving the co-creation of the service experience (rides and attractions) leading to an outcome (entertainment and pleasure). As in the current work, they recognise the limitations of DES alone for modelling human movement in service systems. For their model they adopt a DES package (AutoMod) and use its facilities to introduce an agent-based approach. The model represents customer perceptions, decision-making and movement, and it aims to answer questions about the quantity and location of signs, maps and informed employees in a theme park.

The second example is described by Garnett (2008). Coming from a DES world, he adopts an ABS approach for modelling queuing systems and demonstrates it with an aircraft boarding model. The approach represents a system as a grid with a set of service points and queues. Customers and service staff are represented as agents. The model includes agent perceptions and logic for agent movement and queuing. It is proposed that the model can be used, among other things, for investigating the layout of queuing systems.

Finally, Ustan et al (2006) focus on modelling of physical security systems in retail outlets, including the modelling of regular customers, shoplifters and security personnel. The aim is to design and analyse a security system, along with its protocols and policies, in order to improve the level of protection for the retail outlet under investigation. As part of this work the authors describe a conceptual architecture for defining the environment and incorporating spatial features for visual cognition within that environment (Ustan et al, 2005). This architecture adapts a DES approach to model spatial features and spatial behaviour. Although this work is not directly aimed at improving customer service the architecture has features that are relevant to modelling service operations.

\subsection{Modelling Human Movement}

Related to service operations layout modelling there is the related area of modelling human movement in a built environment. In particular simulations have been applied to modelling evacuation from buildings and pedestrian flow. Both of these involve elements of human 
movement, interaction and decision-making as is required for service operations modelling where the customers are present.

Emergency evacuation models often represent the evacuation space as a grid. Lo et al (2004), for instance, describe the SGEM package (spatial-grid evacuation model). This converts a computer-aided design of a building into a network with a series of nodes (an enclosed space with at least one exit). The movement of evacuees is simulated via movement across a grid that is generated within each node. Thompson and Marchant (1995a) describe the SIMULEX model which aims to model the evacuation of large populations from complex buildings. They use a coordinate-base approach, rather than a grid, arguing that this has particular benefits in terms of treating each person as an individual and in improving the precision of the model (Thompson and Marchant, 1995b). Kim et al (2004) describe how the rules of physics can be used to determine the movement of evacuees from a marine vessel. Brailsford and Stubbins (2006) use DES for modelling evacuation from a building. The model references data on egress time, generated from a social force simulation, depending on the position of an evacuee and the number of people in that area of the building. Chen and Zhan (2008) adopt an ABS approach when modelling the evacuation of urban residents in response to an emergency. They model individual vehicles and their interactions with one another during an evacuation.

In pedestrian flow modelling Hanisch et al (2003) identify a number of ways of modelling pedestrian flow:

- Macroscopic: using ideas from fluid dynamics

- Mesoscopic: where individuals are formed into groups with specific behaviours

- Microscopic: where each person is represented by an entity, or through cellular automata, or with multi-agent-based modelling

Such models are used for evacuation modelling, but are used more generally for investigating the flow of people in public buildings, for instance, a subway station (Bauer et al, 2007), and in complex environments based on an abstract map of the environment (Koh and Zhou, 2011). 
There is commercial software for modelling pedestrian flow. Legion (www.legion.com accessed February 2014) uses a microscopic approach for modelling pedestrian flow. Examples show it being used for modelling the movement of people in transportation facilities (e.g. airports and bus terminals) and at sports events. It is also used for evacuation modelling. The AnyLogic software (www.anylogic.com accessed February 2014) includes a 'pedestrian library' that models the flow of pedestrians in the physical environment. Meanwhile, the Urban Analytics Framework (www.crowddynamics.com/products/uaf.php accessed February 2014) appears to provide similar facilities and includes the modelling of traffic flow as well as pedestrians. There does not, however, appear to have been much use of these tools by those working in operational research; at least not in the published literature. The author is aware that Legion has been used by operational research workers at Transport for London. In a recent article in the operational research literature on modelling vehicle flow and building design, the authors opted to use a DES approach in favour of an ABS pedestrian flow modelling tool, but they do not provide any reasoning for their choice (Otamendi et al, 2012).

There are clearly a number of ideas above that could be used for modelling the movement of humans and service delivery within a service operation in which the customers are present. In this paper the approach used is similar to that of Garnett and Lo et al, that is, entities moving on a grid. Microscopic modelling was seen as most applicable to the situation we are trying to simulate since it enables individuals to make decisions, to interact and to be tracked through the system.

\section{A COFFEE SHOP MODEL}

An adapted DES approach for modelling a service operation in which the customers are present is now demonstrated through a model of a simple coffee shop which has been developed using Excel/VBA. The coffee shop is named the 'Flat White' after a type of coffee served in New Zealand where the model was written during a period of sabbatical leave. The structure of the model is described below. A copy of the Excel/VBA implementation of the model is available from the author upon request. 


\subsection{Basic Structure of the Model}

The model is developed around a 50 x 50 grid. Each cell represents the area taken up by a human entity. The grid represents the area available for placing the facilities required in the coffee shop, in this example: an order point, collection point, five tables and a WC. A user can place these facilities wherever he/she likes within this space. The customer entities will then move across the grid, using the shortest path, in order to receive the service they require. On receiving a service, the customer entities choose where to move to next.

Apart from defining a customer entity, the only other components of the model are the facilities listed above. There are no queues defined in the model, since queues are emergent properties from the entity movements and the design of the coffee shop. An entity can be identified as queuing when it attempts to move but its path is blocked by another entity. As such, the total time an entity spends queuing is the total time an entity spends in a blocked state. Resources (e.g. service staff) could be defined as intelligent entities (agents), but for now they have been left out of the model.

The simulation uses a standard DES three-phase executive (Paul, 1993; Pidd, 2005; Tocher and Owen, 2008). This manages the simulation through a list of future events. In the first (A) phase, the next event is identified; in the second (B) phase that event is executed; in the third (C) phase further events are executed that result from the change in the state of the system instigated in the B-phase. In this model the bound (B-phase) events are: entity arrives, move entity one grid space, end an activity and finish the simulation run. The conditional (C-phase) events are: unblock entities (required to move entities forward once they have been unable to move because, for instance, they are in a queue), entity exits the model and entity reached location. The latter is a point at which the entity cedes control to the system and the service point determines when the entity is free again to move on completion of the service.

As stated above, the customer entities move according to a simple shortest path algorithm. The shortest path is determined by comparing the ratio of the change required in $x$-coordinate and $y$-coordinate at the start of the sequence of moves, to the ratio of the change required in $x$-coordinate and $y$-coordinate given an entity's current position. The algorithm maintains the 
current ratio $(C R)$ close to the rounded value of the starting ratio $(S R)$. The starting ratio is calculated as:

$$
S R=\frac{D(x)-S(x)}{D(y)-S(y)}
$$

where $D$ is the destination coordinates (x and y) on the grid and $S$ is the starting coordinates ( $x$ and $y$ ). The current ratio is:

$$
C R=\frac{D(x)-C(x)}{D(y)-C(y)}
$$

where $C$ is the current coordinates (x and y) of the entity. The changes in $x$ and $y$ for the next move are then calculated as:

$$
\Delta x=\frac{D(x)-C(x)}{|(D(x)-C(x))|}, \quad \Delta y=\frac{D(y)-C(y)}{|(D(y)-C(y))|}
$$

This will yield values of 1 or -1 for $\Delta x$ and $\Delta y$. In order to maintain the current ratio $(C R)$ close to the starting ratio (SR), the values of $\Delta x$ and $\Delta y$ are set to 0 for some moves, creating vertical and horizontal moves respectively.

As an example, if the start position of the entity is $(20,20)$ and it is moving to grid position $(44,10)$, then its start ratio $S R=(44-20) /(10-20)=-2.4$, which is rounded to -2 . If the entity's current position is $(28,18)$, then the current ratio $C R=(44-28) /(10-18)=-2$. Because $C R=$ $S R$, we use the formula above for $\Delta x$ and $\Delta y$, making the next move $(1,-1)$ and the entity moves to grid position $(29,17)$. For the next move $C R$ is now -2.143 . Since $C R<S R$, the entity is deemed to be slightly off course. In order to maintain the course of the entity, $\Delta x$ is calculated as before and set to 1 , but $\Delta y$ is set to 0 . The entity moves to grid position $(30,17)$ and the value of $C R$ returns to -2 .

Further to this shortest path algorithm, the entities avoid head on collisions with other entities and they have some limited capacity to avoid obstacles (denoted in black) on the grid. This is achieved by an entity attempting to make a move to an adjacent grid position, other than that desired by the shortest path, when faced with either of these conditions. The potential to use more sophisticated algorithms for agent moves and collision avoidance is discussed in section 5.3 . 


\subsection{Input Data}

The data required for the model is split into data concerning the coffee shop system and data concerning the customer entities. For the coffee shop system the user defines the coordinates of the entrances and exits; there are two of each in the Flat White model. The only other system data required are for the service points: name, coordinates and mean service time (which is assumed to follow a negative exponential distribution). The user can draw 'obstacles' in suitable locations on the grid by filling cells in black.

For the customer entities, the data defines the mean inter-arrival time (again assumes negative exponential) and the speed with which each entity travels (defined as the time to move one cell). The proportion of entities that travel at different speeds is defined in the data and sampled when the model runs. Customer entities are also split according to their residency time at the tables. In the current model this is represented by 70 percent of customers spending a 'short' time at the tables (mean $=15$ minutes) and 30 percent spending a long time (mean $=30$ minutes). This represents different intentions, for instance, business people who use the coffee shop for access to the internet spend longer at the tables.

Further heterogeneity among the customer entities is generated by sampling their intentions when entering the model. These intentions are defined as a sequence of activities to be completed and so they define the entities route through the service system. The intentions used in the Flat White model are shown in table 1. The entity intentions are sampled according to the proportions shown in column 1 of the table. The model represents some scope for changing intentions based on the state of the system. If all the tables are occupied when a customer entity is trying to sit at one, then the entity will change its intention and leave the coffee shop.

Table 1 Customer Entity Intentions in the Flat White Model

\begin{tabular}{cccccc}
\hline Proportion & Stage 1 & Stage 2 & Stage 3 & Stage 4 & Stage 5 \\
\hline 0.1 & Order & Collect & Table & WC & Exit \\
0.6 & Order & Collect & Table & Exit & \\
0.3 & Order & Collect & Exit & & \\
\hline
\end{tabular}


Greater levels of heterogeneity could be added to the model by including further attributes such as aggressiveness (barging in front of people) and patience (willingness to wait in a queue). This requirement is discussed further in section 5.3.

\subsection{Model Display}

Figure 2 shows the display of the model. The clock in the top left corner shows the current simulation time in units specified by the user; here it is minutes. The location of customer entities is shown by cells coloured in light grey. Note that there are small queues forming at the order and collection points. The cells coloured in black represent obstacles and they are used to show the layout of the coffee shop.

Figure 2 The Flat White Model Simulation Display

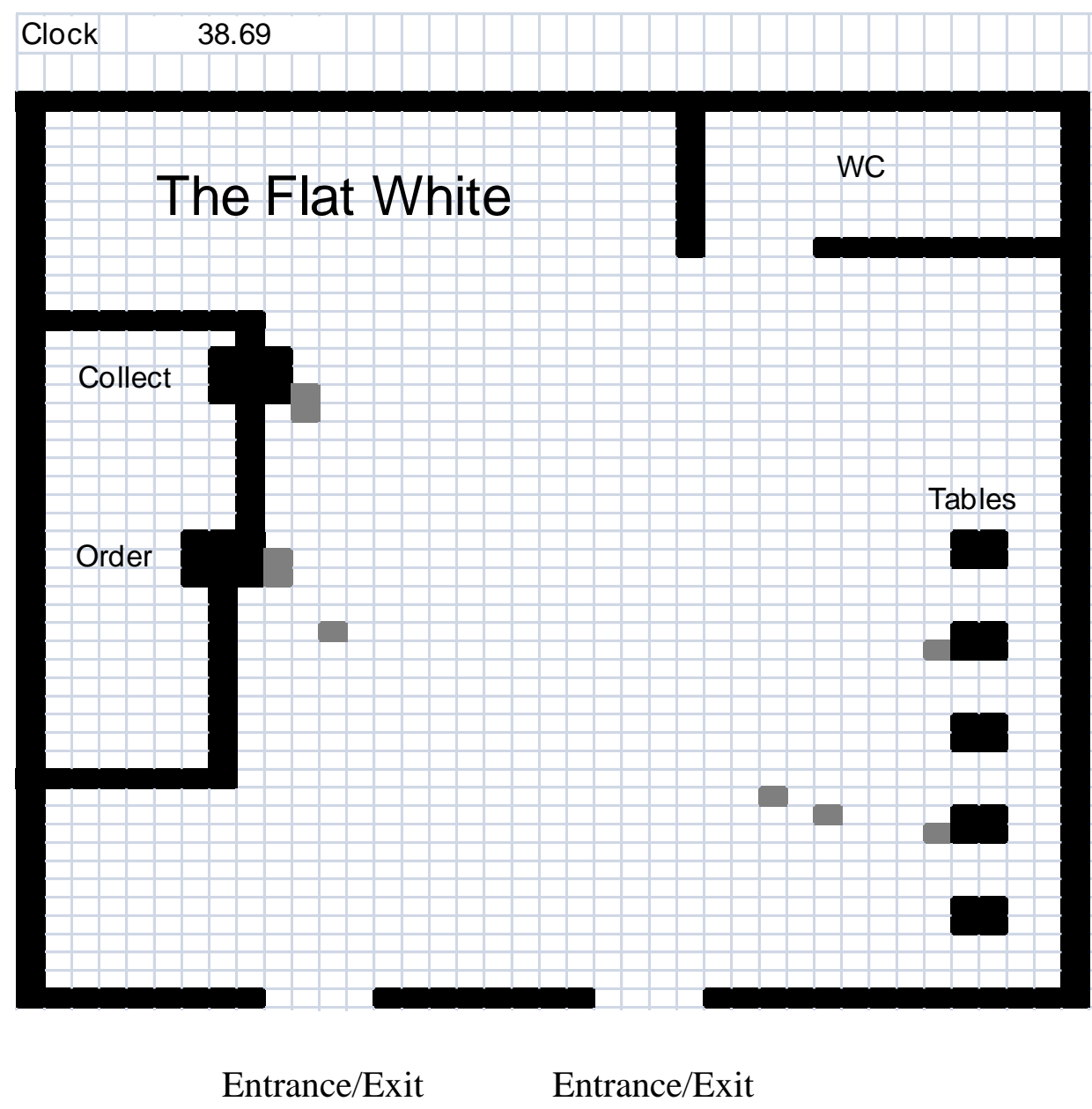




\subsection{Results}

Results are collected for the number of customers served, the number that changed their intentions, the time customer entities spend in the system (split into in service, moving and queuing) and the utilisation of the service points. Other results could be collected if desired, for example, the distance that entities travel.

Table 2 shows the results generated by the model from ten independent replications of 480 minutes, which equates to a working day. The mean, standard deviation and 95\% confidence intervals are given for each value. For comparative purposes the results given in table 3 are generated from a second layout for the coffee shop which is shown in figure 3 .

Table 2 Results from 10 Independent Replications of 480 Minutes with the Flat White Model (Figure 2)

\section{Customers served \\ Change intentions}

Time in system (mins)

Mean

Min

Max

In service (mins)

Moving (mins)

Queuing (mins)

\begin{tabular}{rccc} 
& & \multicolumn{2}{c}{ 95\% confidence interval } \\
\cline { 3 - 4 } Mean & $\begin{array}{c}\text { Standard } \\
\text { deviation }\end{array}$ & Lower & Upper \\
\hline 113.60 & 9.81 & 106.58 & 120.62 \\
9.70 & 5.72 & 5.61 & 13.79
\end{tabular}

Activity utilisation (\%)

Order

Collect

Table 1

Table 2

Table 3

Table 4

Table 5

WC
14.61

1.08

110.11

12.86

1.34

0.41

24.79

12.32

74.09

65.55

59.70

45.58

36.27

5.67
0.88

0.16

32.69

0.79

0.05

0.11

3.62

1.88

6.34

7.10

8.12

10.33

9.90

2.48
13.98

0.96

86.72

15.23

1.19

133.49

13.43

1.30

1.38

0.49

0.33

27.39

13.67

78.62

69.56

70.63

60.47

65.51

53.89

52.96

38.19

43.35

29.19

7.45 
Table 3 Results from 10 Independent Replications of 480 Minutes with the Flat White Model Alternative Layout (Figure 3)

Customers served

Change intentions

\begin{tabular}{rccc} 
& & \multicolumn{2}{c}{ 95\% confidence interval } \\
\cline { 3 - 4 } Mean & $\begin{array}{c}\text { Standard } \\
\text { deviation }\end{array}$ & Lower & Upper \\
\hline 113.40 & 9.66 & 106.49 & 120.31 \\
8.90 & 4.53 & 5.66 & 12.14
\end{tabular}

Time in system (mins)

Mean

Min

$\operatorname{Max}$

In service (mins)

Moving (mins)

Queuing (mins)

$\begin{array}{rr}14.85 & 0.80 \\ 1.28 & 0.19 \\ 110.78 & 31.46 \\ & \\ 12.82 & 0.80 \\ 1.63 & 0.05 \\ 0.39 & 0.11\end{array}$

14.28

1.14

88.27

15.42

1.41

133.29

Activity utilisation (\%)

Order

24.72

12.69

75.02

Table 1

Table 2

69.64

54.78

Table 3

Table 4

Table 5

45.37

35.33

WC

3.81

2.28

3.61

6.94

9.13

5.84

12.94

2.59
12.25

1.60

0.31

13.40

1.67

0.47
21.99

11.06

72.43

64.68

48.25

41.19

26.07

3.82
27.45

14.32

77.60

74.61

61.31

49.55

44.59

7.53 
Figure 3 Alternative Layout for the Flat White Simulation

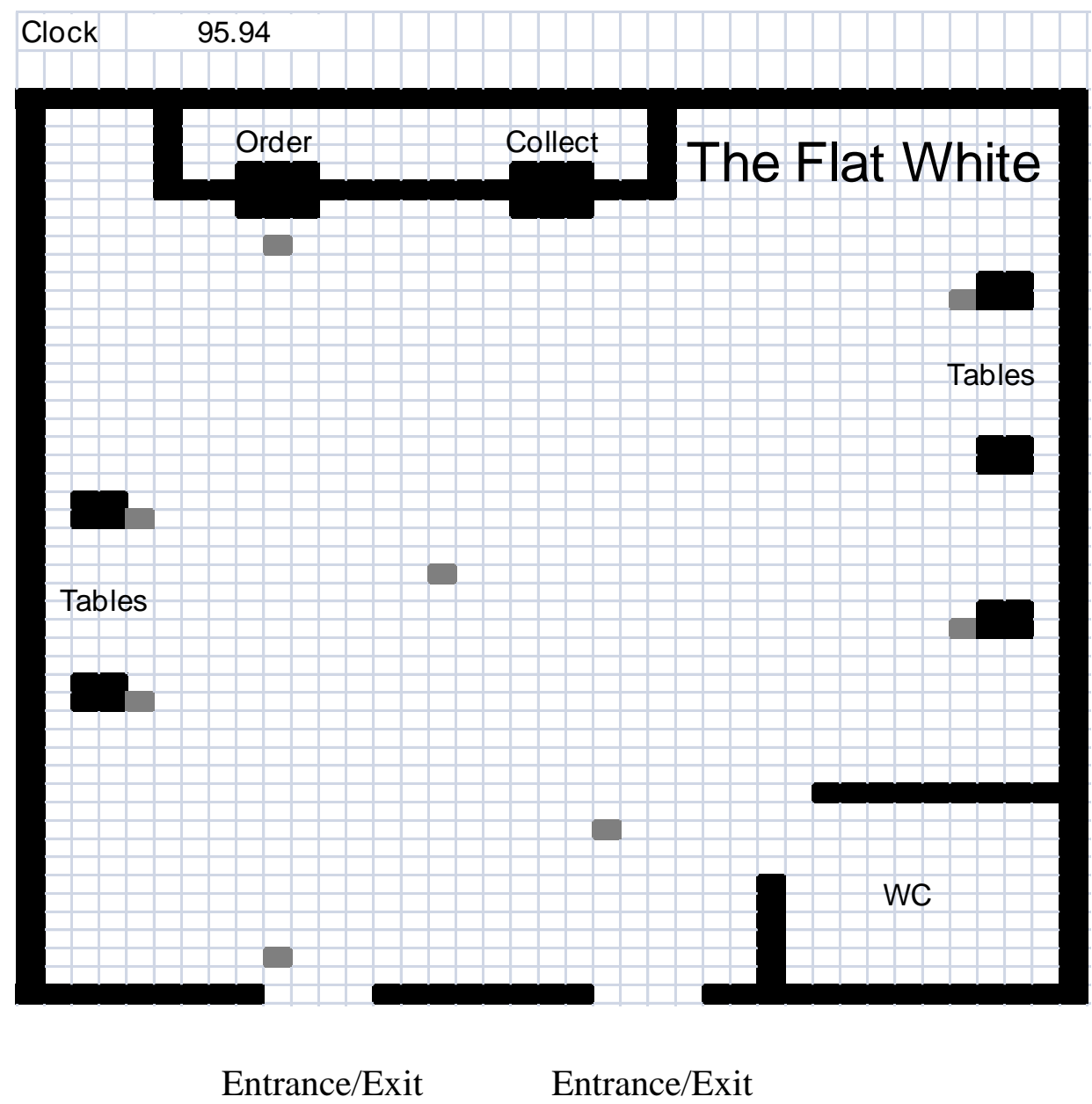

The results from the two layouts demonstrate similar levels of performance for most of the statistics, as shown by the overlapping confidence intervals. However, the mean time spent moving appears to be greater for the second layout; 1.63 minutes as opposed to 1.34 minutes for the first layout. This is confirmed by a paired- $t$ confidence interval for the differences between the two results at five percent significance $(-0.33,-0.27)$. The paired- $t$ interval is used because the model induces correlation between the results of the two layouts through the use of common random numbers (Law, 2007). This difference in time spent moving results from the greater distances customers must walk in the second layout due to the configuration of the coffee shop area. If we assume that customers would prefer to spend less time moving around the coffee shop, then the original layout in figure 2 is the better option. As such, the model provides the facility to compare the performance of different layouts for the coffee shop. 
The impact of different customer behaviours can also be predicted from the model. For instance, the model is run with 50 percent of customer entities having a 'long' residence period at the tables, instead of only 30 percent. The results from ten replications demonstrate an increase in table utilisation and time in the system. Meanwhile, more customers change their intentions and decide to leave the coffee shop rather than wait for a table. In the first layout (figure 2), the mean number of customers that change their intentions increases from 9.70 to 12.90 , which is a significant difference at the five percent level. This suggests there will be a lower level of satisfaction, in terms of more customers changing their intentions, if a greater number of customers choose to spend longer at the tables.

Finally, the model is able to provide time-based results. Figure 4 shows an example of such a graph showing how the number of customers in the system varies over time in the original layout. Such data can be useful for identifying specific patterns that result from the behaviour of the customer entities in the system. Here we note that there are never more than eight customers in the coffee shop, which is partially a result of customers not waiting for tables.

Figure 4 Number of Customers in the System over Time: Original Layout (Figure 2), Replication 1

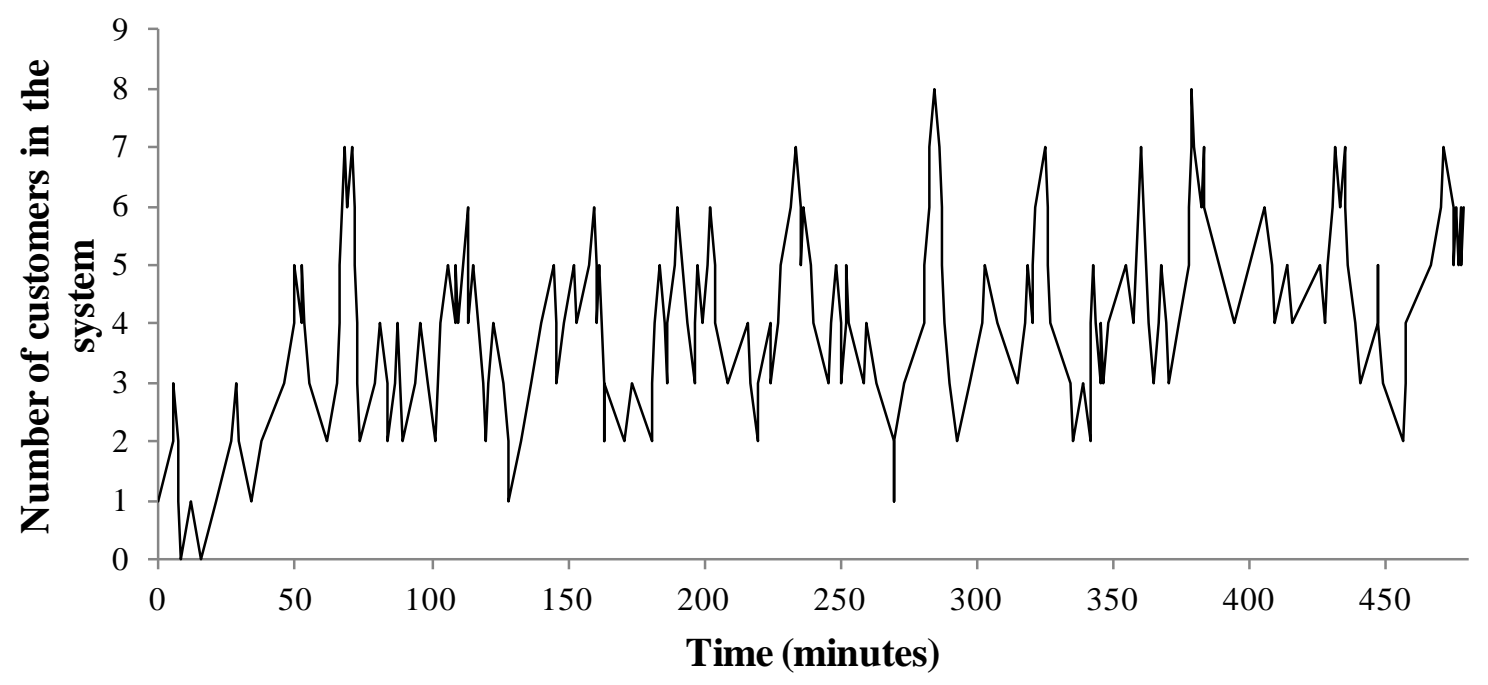

\section{DISCUSSION}

Having presented the model we now discuss the key differences between the Flat White model and previous modelling of service systems in which the customers are present. We 
then ask if the Flat White model is, in fact, an ABS, and we explore possible extensions to the modelling approach.

\subsection{Differences and Benefits in the Modelling Approach}

The key difference between the Flat White model and the typical DES model of a service system is that the only components defined in the model are entities and activities (facilities). There is no explicit modelling of queues. Instead the queues emerge as a result of the interaction of the entities with the system and with each other. This has the benefit of simplifying the modelling of the system by reducing the number of components that need to be modelled and the associated logic that is required.

The model differs in two other respects. First, the model is spatially aware since the placing of service points on the grid affects the flow of the entities. Second, the entities have some intelligence; they move according to their own intentions and they are able to avoid obstacles and other entities while moving. This level of intelligence is relatively low, but it could easily be extended. This is something that we discuss section 5.3. The benefit of the model being spatially aware and of modelling entity intelligence is that changes to the layout of the system make a difference to its performance. As a result, alternative layouts can be compared.

The Flat White model does have some similarities with Garnett's (2008) ABS queuing model in that it is motivated by a desire to help with layout decisions for service systems and it does so by modelling the system on a grid. However, it does differ in three key respects: it uses a three-phase executive, entities move at different speeds (this is enabled through the use of a DES executive) and the absence of queues as defined components of the system. Meanwhile, Garnett implemented his model with more intelligent agent behaviour including, for instance, field of vision and ability to anticipate future events. This was aided by using standard ABS software (AnyLogic and NetLogo). Given that there is some level of correspondence with Garnett's ABS model, we now explore whether the Flat White model is in fact an ABS. 


\subsection{Is the Flat White Model an Agent-Based Simulation?}

The origins of ABS lie in the desire to study complex (adaptive) systems and their emergent behaviours (Heath and Hill, 2010). The approach, that was popularised by the Sante Fe Institute through its Swarm software (www.swarm.org accessed February 2014), has been applied across a wide range of fields for studying biological, physical and social systems, for instance. The basic idea is to model systems from the bottom-up as a set of agents, with individual behaviours, that interact over time. The aim of modelling systems in this way is to observe the behaviours, patterns and structures that emerge (Macal and North, 2010).

Macal and North (2010) describe the structure of an ABS model as consisting of three elements:

- Agents: with attributes and behaviours

- Agent relationships: defining who agents interact with and how

- Agent environment: the environment in, and with, which the agents interact

Schelling's model of segregation is an early example of ABS (in this case not on a computer) in which the dynamics of a population that is split into two groups who aim for a desired level of segregation is investigated (Schelling, 1971). The model demonstrates that a much higher level of segregation is typically achieved than the desired level. Axelrod (1997) outlines a number of ABS models which he uses to study phenomena in the arena of politicalscience. One such model investigates how cultures spread and how they remain distinct over time (chapter 7).

In a similar vein to Axelrod's cultural model, Deffuant et al (2002) describe a model of opinion dynamics which they use to model the influence of extremists in a population. The key difference to Axelrod's model is that opinions are seen as continuous rather than discrete variables. Meanwhile, Parker and Epstein (2012) use ABS for modelling epidemics. Using distributed computing their model is able to represent the outbreak of an epidemic in a population with billions of agents. 
These examples of ABS models are used to test the theories of the phenomena that are under investigation. They bear little relation to our context which is more empirically driven (based on data that can be observed in a real system) and aimed at aiding specific decisions. However, as ABS adoption has spread so we see examples of empirically driven models that are used to aid decision-making. For instance, Macal and North (2005) discuss the validation of such a model (EMCAS: Electricity Market Complex Adaptive System) which was designed to aid decision-making around restructuring and deregulation of electricity power markets. Meanwhile, Edmunds et al (2006) discuss the collection of data on human mixing patterns which could aid the development and parameterisation of models of close-contact infectious disease spread. For a detailed survey of recent examples of ABS modelling see Heath et al (2009).

Having briefly introduced the concepts of ABS, we return to our focus here which is to classify whether the Flat White model is either a DES or an ABS. However, the problem we encounter in adopting such a polarised classification is that these two terms are referring to quite different facets of the respective modelling approaches. 'Discrete-event simulation' is referring to the time-handling mechanism used to move from event-to-event. This results in an irregular time-step. The term 'agent-based simulation' is describing the modelling of individual intelligent agents that interact and it is from these interactions that the overall system behaviour emerges.

If we dig a little deeper, then we find that DES generally takes a top-down view of the world. That is, the system is designed and this regulates the activities of the entities that move around the system. The use of attributes for entities gives them some control of their destiny, but again it is generally the system that uses this information to determine the next action for an entity, i.e. the entities are not intelligent. DES models also focus on modelling the interaction of random events, which can lead to surprising system behaviour. Given that DES emerged from manufacturing (Tocher, 2008), these facets are reasonable representations of that world.

ABS takes a bottom-up view of the world. At an extreme this means the system is not designed, but that it emerges from the behaviour and interaction of intelligent agents. Time is often modelled as a fixed time step which describes the period between either possible interactions between agents or agent decisions. Randomness can be included in these 
models, but it tends to be restricted to modelling probabilities of events (e.g. an interaction being successful) and not for modelling activity times (which is the prime use of randomness in DES). Table 4 summarises these facets of DES and ABS.

Table 4 Facets of DES and ABS Modelling

\begin{tabular}{lll}
\hline \multicolumn{1}{c}{ Facet } & \multicolumn{1}{c}{ DES } & \multicolumn{1}{c}{ ABS } \\
\hline World view & Top-down & Bottom-up \\
Time handling & Irregular time step & Fixed time-step \\
Randomness & Key facet of model & Generally used only for \\
& Represents times and & probabilities \\
& probabilities & \\
\hline
\end{tabular}

Applying these facets to the situation of modelling the layout of a retail outlet, and other types of service system, we should recognise the following. A retail outlet is designed in the sense that an organisation determines where resources will be placed. This suggests a topdown view. On the other hand, intelligent agents (customers) arrive into the designed system where they interact with that system and with one another. This suggests a bottom-up view. The emergent behaviour is a result of both the system design and the interaction of the intelligent agents. Agents maintain control of their activities while moving around the designed system, but cede control to that system when receiving service. The time to perform activities (e.g. service times) will be subject to random variation. All this suggests a mix of the facets listed in table 4. Hence, we would argue that the Flat White model is not a pure DES, but a mixed DES/ABS.

Of course, some would argue that the model is strictly a DES, since it simply represents a system as a set of entities with limited intelligence and time progresses using a standard DES executive. However, with an extension to the intelligence of the entities (as discussed in the next section), the argument that the model involves at least some elements of ABS would be strengthened much further. Meanwhile, from a pure ABS perspective it might be argued that the design of the physical system is not exclusively a facet of DES modelling. Indeed, the physical system could be interpreted as being the agent environment. The change from agent 
control to system control as an agent progresses through the environment does still imply, however, that there are elements of a DES worldview in the model.

The debate over whether the Flat White model is an ABS or a DES depends on whether we consider the two simulation approaches to be strictly separable, or we consider that there is a continuum from pure DES to pure ABS with many shades in between. For instance, we might argue that as soon as an entity in a DES model has an attribute whose value impacts on the logic of the model then we have made a first move towards a partial ABS approach. For a useful discussion on these issues, see Brailsford (2014).

\subsection{Developing the Flat White Model Further}

The adapted DES model has demonstrated two key benefits in modelling service systems in which the customers are present: it simplifies the modelling and it can aid decisions about layout as well as the number of resources. The Flat White model is a relatively simple representation of a small scale service system. There is much potential to develop the idea further.

A key area that needs to be developed further is the intelligence of the customers. This could entail how the customers make decisions, how they react to the environment and how they move through the environment. The model currently assumes customers are rational, e.g. they find the shortest route from their present location to their intended destination. This may be a reasonable assumption for movement, but not necessarily for all decisions in a service system. For instance, how does a person decide which route to take around a supermarket? It is unlikely that this is a perfectly rational decision, especially in the light of limited information on the layout of the supermarket. We know humans are subject to bounded rationality (Simon, 1957). It may be useful to model the limits of information available to people such as the effect of signs and line-of-sight. Further, a more sophisticated algorithm could be employed for finding a route from one location in the service system to another; for instance, the $\mathrm{A}^{*}$ algorithm (Hart et al, 1968).

The model includes simple collision avoidance for both other people and obstacles, but this could be extended to include a more sophisticated approach. The customers are also given a fixed set of intentions, except that they may choose to leave if all the tables are occupied. 
More sophisticated changes to their intentions could be modelled as well as ambiguous intentions (being unsure about what to do next).

The key to developing the customer intelligence element of the model may be in working with standard models of human decision-making and pedestrian behaviour. In simulation the PECS (physical conditions, emotional state, cognitive capabilities and social status) architecture has been used for modelling human behaviour (Schmidt, 2000; Brailsford and Schmidt, 2003). The BDI (belief-desire-intention) model has also been adopted in simulated environments (Lee and Son, 2009; Padgham et al, 2011). Brailsford et al (2012) used the theory of planned behaviour (Ajzen, 1991) to model the behaviour of women in deciding whether to attend for breast cancer screening. The work of Helbing et al $(2001,2005)$ may prove particularly useful for modelling the movement of individuals in the service environment, albeit that their work focuses primarily on pedestrian movement.

Given that such models are applicable, then it may be possible to apply a standard set of data for customer behaviour in a family of service operations such as a coffee shop or supermarket. However, the collection and categorisation of these data is a major challenge.

Another extension is to implement intelligent resources in the model. Service staff have their own intentions and behaviours. The movement of staff and their interaction with the customers will affect the performance of the service operation.

The results generated by the model in its present form are largely typical of those generated by a DES model of a service system. Extending the results to reflect the customers' experience in the system would provide additional insight into the relative merits of alternative layouts. For instance, data could be collected on the extent of obstacle and collision avoidance as a means for reflecting the sense of space a layout provides. Reducing the need to avoid obstacles and collisions would generally seem to be beneficial to the customers' experience of a service.

The use of a DES executive for modelling customer movement needs to be investigated further. This may significantly slow the model when there are many customers in the system. Use of a global time-step, as is typical in many ABS models, may improve the speed of the 
model, but may also result in loss of accuracy, particularly as this forces all customers to move and make decisions at fixed time intervals.

\section{CONCLUSION}

This paper presents a set of ideas on how to develop models of service operations (in which the customers are present), when the aim is to help determine the layout of the operation. The key innovation is to be able to model a service system without explicitly defining queues. The approach is arguably a mixed DES/ABS model. With the implementation of a set of extensions, this approach could prove extremely valuable in a live decision-making environment.

Overall, the aim is to develop an easy-to-use modelling environment where a user can dragand-drop activities and obstacles onto a grid, and enter data about the system being modelled. This requires both careful software design and implementation of standard data sets for modelling human behaviour. It is the latter which poses the greatest challenge for this approach.

There is clearly scope for further research in developing the approach and the methodology for applying it in a real context. The key areas for further research into this approach are: developing the intelligence of the customer entities; data collection and generating standard data sets for a modelling context; adding intelligent resources to the modelling approach; exploring results that reflect the customer experience of the system; and determining the best simulation executive for supporting this approach. With such research, the approach could provide a straightforward means for modelling and improving service operations in which the customers are present.

\section{ACKNOWLEDGEMENTS}

The author acknowledges the helpful input over the years of Masters project students who have worked on this problem from various perspectives: Annie Chan, Miranda Luk, Hayley Souter, Man-Fung Wong and Aikaterini Drosou. 
This paper is adapted and extended from: Robinson, S. (2010). Modelling Service Operations: A Mixed Discrete-Event and Agent Based Simulation Approach. Proceedings of the $5^{\text {th }}$ Simulation Workshop (SW10) (Gunal, M., Tjahjono, B., Robinson, S. and Taylor, S.J.E., eds.), Operational Research Society, Birmingham, pp. 192-199.

\section{REFERENCES}

Ajzen, A. (1991). The Theory of Planned Behaviour. Organizational Behaviour and Human Decision Processes, 50, 179-211.

Axelrod, R. (1997). The Complexity of Cooperation: Agent-Based Models of Competition and Collaboration. Princeton, New Jersey: Princeton University Press.

Bauer, D., Seer, S. and Brändle, N. (2007). Macroscopic Pedestrian Flow Simulation for Designing Crowd Control Measures in Public Transport after Special Events. Proceedings of the 2007 Summer Computer Simulation Conference, SCS: San Diego, CA, 1035-1042.

Beck, A. (2011). Modelling Passenger Flows in Heathrow Terminal 5. Journal of Simulation, $5,69-76$

Brailsford, S.C. (2014). Discrete-Event Simulation is Alive and Kicking. Journal of Simulation, 8 (1), 1-8.

Brailsford, S.C., Harper, P.R. and Sykes, J. (2012). Incorporating Human Behaviour in Simulation Models of Screening for Breast Cancer. European Journal of Operational Research, 219, 491-507.

Brailsford, S. and Schmidt, B. (2003). Towards Incorporating Human Behaviour in Models of Health Care Systems: An Approach using Discrete Event Simulation. European Journal of Operational Research, 150 (1), 19-31.

Brailsford, S. and Stubbins, D. (2006). Using Discrete-Event Simulation to Model Emergency Evacuation of a Public Building. Proceedings of the Operational Research Society Simulation Workshop 2006 (SW06) (J. Garnett, S. Brailsford, S. Robinson and S. Taylor, eds.) Operational Research Society: Birmingham, UK, 45-50. 
Chen, X and Zhan, F.B. (2008). Agent-Based Modelling and Simulation of Urban Evacuation: Relative Effectiveness of Simultaneous and Staged Evacuation Strategies. Journal of the Operational Research Society, 59 (1), 25-33.

Deffuant, G., Amblard, F., Weisbuch, G. and Faure, T. (2002) How can Extremism Prevail? A Study Based on the Relative Agreement Interaction Model. Journal of Artificial Societies and Social Simulation, 5 (4).

Dubiel, B. and Tsimhoni, O. (2005). Integrating Agent Based Modeling into a Discrete Event Simulation. Proceeding of the 2005 Winter Simulation Conference (M. E. Kuhl, N. M. Steiger, F. B. Armstrong, and J. A. Joines, eds.). IEEE, Piscataway, NJ, 1029-1037.

Edmunds, W.J., Kafatos, G. Wallinga, J. and Mossong, J.R. (2006). Mixing Patterns and the Spread of Close-Contact Infectious Diseases. Emerging Themes in Epidemiology, 3 (10).

Garnett, J. (2008). A Cellular Agent Based Method for Modelling Human Queueing Systems. Proceedings of the Operational Research Society Simulation Workshop 2008 (SW08) (C. Currie, K. Kotiadis, S. Robinson and S. Taylor, eds.) Operational Research Society: Birmingham, UK, 43-52.

Hanisch, A., Tolujew, J., Richter, K. and Schulze, T. (2003). Online Simulation of Pedestrian Flow in Public Buildings. Proceedings of the 2003 Winter Simulation Conference (S. Chick, P. J. Sánchez, D. Ferrin, and D. J. Morrice, eds.), IEEE: Piscataway, NJ, 1635-1641.

Hart, P.E., Nilsson, N.J. and Raphael, B. (1968). A Formal Basis for the Heuristic Determination of Minimum Cost Paths. IEEE Transactions on Systems Science and Cybernetics, 4 (2), 100-107.

Heath, B., Hill, R. and Ciarallo, F. (2009). A Survey of Agent-Based Modeling Practices (January 1998 to July 2008). Journal of Artificial Societies and Social Simulation, 12 (4) 9 (http://jasss.soc.surrey.ac.uk/12/4/9.html accessed February 2014).

Heath, B.L. and Hill, R.R. (2010). Some Insights into the Emergence of Agent-Based Modelling. Journal of Simulation, 4 (3), 163-169. 
Helbing, D., Molnär, P., Farkas, I.J. and Bolay, K. (2001). Self-Organizing Pedestrian Movement. Environment and Planning B: Planning and Design, 28, 361-383.

Helbing, D., Buzna, L., Johansson, L. and Werner, T. (2005). Self-Organized Pedestrian Crowd Dynamics: Experiments, Simulations, and Design Solutions. Transportation Science, 39, $1-24$.

Johnston, R., Clark, G. and Shulver, M. (2012). Service Operations Management: Improving Service Delivery, $4^{\text {th }}$ ed. Pearson Education, Harlow, UK.

Kim, H., Park, J.-H., Lee, D. and Yang, Y.-s (2004). Establishing Methodologies for Human Evacuation Simulation in Marine Accidents. Computers \& Industrial Engineering, 46 (4), 725-740.

Koh, W.L. and Zhou, S. (2011). Modeling and Simulation of Pedestrian Behaviors in Crowded Places. ACM Transactions on Modeling and Computer Simulation, 21 (3), 1-23.

Law, A.M. (2007). Simulation Modeling and Analysis. (4 ${ }^{\text {th }}$ ed.). New York: McGraw Hill.

Lee, S. and Son, Y.-J. (2009). Dynamics Learning in Human Decision Behavior for Evacuation Scenarios under BDI Framework. Proceedings of the 2009 INFORMS Simulation Society Research Workshop (Lee, L.H., Kuhl, M.E., Fowler, J.W. and Robinson, S., eds.). INFORMS Simulation Society, 96-100.

Lo, S.M., Fang, Z., Lin, P. and Zhi, G.S. (2004). An Evacuation Model: The SGEM Package. Fire Safety Journal, 39, 169-190.

Macal, C.M. and North, M.J. (2005). Validation of an Agent-based Model of Deregulated Electric Power Markets. Proceedings of North American Computational Social and Organization Science (NAACSOS).

Macal, C.M. and North, M.J. (2010). Tutorial on Agent-Based Modelling and Simulation. Journal of Simulation, 4 (3), 151-162. 
Otamendi, F.J., Laffond, J. and Segundo, N.S. (2012). Design of the Drop-off-pick-up Zones in Buildings: The Case of Private Transportation at the Female University of Riyadh. Journal of the Operational Research Society (2012) 63, 122-129.

Padgham, L., Scerri, D., Jayatilleke, G. and Hickmott, S. (2011). Integrating BDI Reasoning into Agent Based Modeling and Simulation. Proceedings of the 2011 Winter Simulation Conference (S. Jain, R.R. Creasey, J. Himmelspach, K.P. White, and M. Fu, eds.), IEEE, Piscataway, NJ, pp. 345-356.

Pagell, M. and Melnyk, S.A. (2004). Assessing the Impact of Alternative Manufacturing Layouts in a Service Setting. Journal of Operations Management, 22, 413-429.

Parker, J. and Epstein, J.M. (2011). A Distributed Platform for Global-Scale Agent-Based Models of Disease Transmission. ACM Transactions on Modeling and Computer Simulation, 22 (1), Article 2.

Paul, R.J. (1993). Activity Cycle Diagrams and the Three Phase Method. Proceedings of the 1993 Winter Simulation Conference (G.W.Evans, M. Mollaghasemi, E.C. Russell, W.E. Biles, eds.). IEEE, Piscataway, NJ, pp. 123-131.

Pidd, M. (2005). Computer Simulation in Management Science. (5 ${ }^{\text {th }}$ ed.). Chichester, UK: Wiley.

Robinson, S. (2001). Soft with a Hard Centre: Discrete-Event Simulation in Facilitation. Journal of the Operational Research Society, 52 (8), 905-915.

Schelling, T.C. (1971). Dynamic Models of Segregation. Journal of Mathematical Sociology, $1,143-186$.

Schmidt, B. (2000). The Modelling of Human Behaviour. Erlangen, Germany: SCS Publications. 
Simon, H. (1957). A Behavioural Model of Rational Choice. In Models of Man, Social and Rational: Mathematical Essays on Rational Human Behaviour in a Social Setting. New York: Wiley.

Thompson, P.A. and Marchant, E.W. (1995a). A Computer Model for the Evacuation of Large Building Populations. Fire Safety Journal, 24, 131-148.

Thompson, P.A. and Marchant, E.W. (1995b). Computer and Fluid Modelling of Evacuation. Safety Science, 18, 277-289.

Tocher, K.D. (2008). Techniques for the Application of Computers to Industrial Simulation. Journal of Simulation, 2 (3), 138-142.

Tocher, K.D. and Owen, D.G. (2008). The Automatic Programming of Simulations. Journal of Simulation, 2 (3), 143-152.

Ustun, V., Yapicioglu, H., Gupta, S., Ramesh, A. and Smith, J.S. (2005). A Conceptual Architecture for Static Features in Physical Security Simulation. Proceedings of the 2005 Winter Simulation Conference (M. E. Kuhl, N. M. Steiger, F. B. Armstrong, and J. A. Joines, eds.). IEEE, Piscataway, NJ, 958-964.

Ustan, V., Yilmaz, L. and Smith, J.S. (2006). A Conceptual Model for Agent-Based Simulation of Physical Security Systems. ACM-SE 44: Proceedings of the 44th Annual Southeast Regional Conference, ACM, 365-370. 